\title{
Residual stresses in induction-hardened gear teeth mapped by neutron diffraction
}

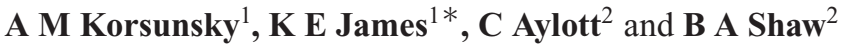 \\ ${ }^{1}$ Department of Engineering Science, University of Oxford, UK \\ ${ }^{2}$ The Design Unit, Department of Mechanical, Materials and Manufacturing Engineering, University of Newcastle upon \\ Tyne, UK
}

\begin{abstract}
Surface-hardening treatments are used in order to enhance the resistance of mating gear teeth surfaces to wear and fatigue crack initiation. The treatments create a compressive residual stress state near the tooth surface, which acts to impede crack initiation and propagation. Accurate determination of this residual stress field is important for quantifying the effect of treatment and optimizing the processing conditions. Neutron diffraction provides a powerful non-destructive experimental tool for residual stress evaluation. By collecting residual strain data for three different orientations, the principal stress directions and values can be mapped. This paper describes the procedure used for building such maps using the G5.2 spectrometer at Laboratoire Léon Brillouin, Saclay, and presents the results obtained for an inductionhardened gear.
\end{abstract}

Keywords: residual stress, diffraction measurement, induction hardening, gears

\section{INTRODUCTION}

Wear and fatigue resistance of gears used for power transmission are application-critical properties. Gear failures are usually classified into several major categories, such as cracking due to root bending, macroscopic wear resulting in gross removal of material from the tooth flank and ultimately the loss of shape and performance, flank micropitting followed by the initiation and growth of a macroscopic fatigue crack, etc. In all cases the performance of a gear is strongly affected by the surface properties [1], such as the precision of the involute tooth shape and the surface roughness, but is also very strongly influenced by the hardness and residual stress state.

In order to enhance the hardness and fatigue resistance of gears, various surface-hardening treatments have been proposed. These include such widely used processes as carburizing, nitriding and carbonitriding, and also induction hardening.

Induction hardening involves rapid heating of the gear tooth surface by a high-frequency electromagnetic field, inducing eddy currents and heat generation, followed by rapid cooling of these regions by heat conduction away from the surface into the body of the gear. As a consequence the near-surface layers undergo a rapid

The MS was received on 14 August 2001 and was accepted after revision for publication on 14 February 2002.

* Corresponding author: Department of Engineering Science, University of Oxford, Parks Road, Oxford OX1 3PJ, UK. quenching heat treatment, which results in the formation of stronger phases, such as martensite, and an increase in the overall hardness of the material. For most hardening treatments the hardening effect is confined to very small depths of several hundred micrometres. However, induction hardening produces significantly deeper penetration, reaching depths of treatment as large as $2-3 \mathrm{~mm}$.

Simultaneously with the phase transformation, the hardening treatment creates a compressive residual stress state near a tooth surface, which acts to impede crack initiation and propagation. In order to be most effective in preventing the initiation and growth of cracks from the tooth flank, the residual stress state must have its most compressive component aligned with the tooth surface. This is clearly the case for the immediate vicinity of the surface, where the stress component normal to the surface vanishes to satisfy the stress-free boundary condition. The principal stress directions are therefore parallel and perpendicular to the surface.

The precise nature of the stress state variation with depth is more difficult to establish. Conventional laboratory $\mathrm{X}$ ray diffraction can be used to measure stress components parallel to the surface. Some success has been achieved in measuring the variation of in-plane stress with distance from the original surface in shot-peened gear teeth [1]. However, because of the very shallow penetration of lowenergy X-rays, the stress component normal to the surface cannot be measured. Stress depth profiling is performed by electrochemical layer removal but, after each stage of etching, the condition of zero normal stress is recreated. 
Thus, if complete stress state characterization is the objective, the use of penetrating radiation is a requirement rather than an option. Accurate determination of the residual stress field is important for quantifying the effect of treatment on durability, and for optimizing the processing conditions.

\section{NEUTRON DIFFRACTION MEASUREMENTS}

Diffraction provides a powerful experimental tool for residual stress evaluation [2]. Compared with X-rays, the penetrating ability of neutron radiation allows sampling volumes to be scanned through the bulk of larger samples [3], up to centimetres in thickness. The strain is determined from the shift in the peak position. For monochromatic radiation of known wavelength $\lambda$, the angular shift $\Delta \theta$ is related to lattice spacing increment through the derivative of Bragg's formula:

$$
\varepsilon=\frac{\Delta d}{d_{0}}=-\cot \theta_{0} \Delta \theta
$$

Determination of strain thus requires knowledge of the undistorted lattice spacing parameter $d_{0}$ for the chosen reflection, which is usually found using an unstressed sample. In this study the reference spacing was found by requiring that the stresses acting in the radial direction balance along a line across the gear tooth. The choice of $d_{0}$ was also substantiated by the fact that the values extrapolated from the maps towards the gear surface matched previous measurements made on induction-hardened gears using laboratory X-rays. The process of induction hardening does not produce any compositional variation, unlike processes such as carburizing. Therefore, no allowance was made for a variation in $d_{0}$ with position due to compositional effects.

In the present study the purpose was to map the principal stress values and orientations in an induction-hardened gear tooth using neutron diffraction on the G5.2 spectrometer at Laboratoire Léon Brillouin, Saclay. This was achieved by collecting residual strain values for three different orientations over a square mesh of points. The neutron beam was monochromated to the wavelength of $2.866 \AA$ using a crystal of pyrolytic graphite. The 110 peak of b.c.c. ferrite was chosen. At this wavelength the corresponding peak was located at the Bragg angle $2 \theta=92^{\circ}$, so that the sampling volume used was an approximately squaresection prism with the dimensions of $1 \mathrm{~mm} \times 1 \mathrm{~mm} \times$ $10 \mathrm{~mm}$ approximately, with the $10 \mathrm{~mm}$ dimension out of the plane of the tooth.

The width of the gear tooth analysed in the present study was about $50 \mathrm{~mm}$. The hardening treatment conditions were uniform across the tooth width, and the measurements were conducted over sampling volume $10 \mathrm{~mm}$ wide, lying in the middle of the tooth width and sufficiently far away from the edge to ignore edge effects. The stress-strain state within the tooth and the measured values obtained under these conditions are well described by the assumption of plane strain. Thus, only two principal strain components had to be determined within the plane of gear rotation. However, since the orientation of these principal strains (and stresses) is not known a priori, it was resolved to measure three components of elastic strain and to reconstruct the complete strain and stress state from those obtained by plane strain analysis. The three strain orientations measured were dictated by the conditions of beam access and path length within the sample, and the ease and accuracy of measurement interpretation.

The measurement of elastic lattice strain by neutron diffraction involves precise determination of the scattering angle produced by the ensemble of grains lying within the sampling volume. If the sampling volume is only partially submerged into the sample, the peak centre can suffer from spurious shift, unless special care is taken. Post-measurement compensation is known to be susceptible to further numerical inaccuracies, while implementing special collimating arrangements is expensive and reduces the counting efficiency. Consequently, in the present study the nearsurface measurements which were likely to be affected by this aberration effect were discarded. The location of the measurement points which contributed to the maps presented below is clearly indicated in the figures with respect to the gear tooth outline.

\section{ANALYSIS}

The strain measurement directions were chosen at $60^{\circ}$ intervals and oriented with respect to the gear tooth as shown in Fig. 1. This allows the three sets of strain measurements to be treated in the same way as the data from the $60^{\circ}$ strain gauge rosette. Elementary strain analysis based on the two-dimensional Mohr's circle [4] allows the strain $\varepsilon_{1}$ to be expressed using the following equation:

$$
\varepsilon_{1}=a+r \cos (2 \alpha)
$$

where

$\alpha=$ angle between the strain direction 1 and the maximum principal strain direction

$a=$ direct strain corresponding to the centre of the circle

$r=$ radius of Mohr's circle

Since the three directions of strain were physically separated by angles of $60^{\circ}$, it holds that, for strains $\varepsilon_{2}$ and $\varepsilon_{3}$,

$$
\begin{aligned}
& \varepsilon_{2}=a+r \cos \left(2 \alpha+120^{\circ}\right) \\
& \varepsilon_{3}=a+r \cos \left(2 \alpha-120^{\circ}\right)
\end{aligned}
$$


(a)

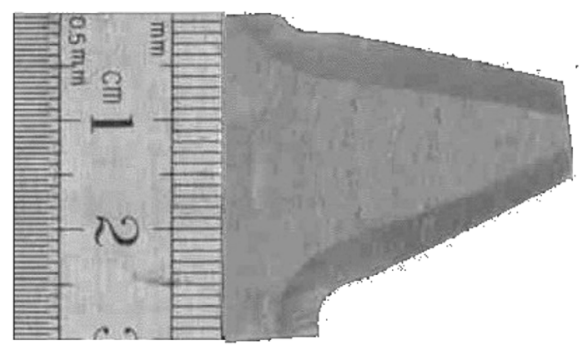

(b)

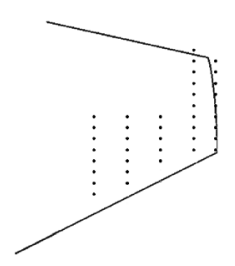

(c)

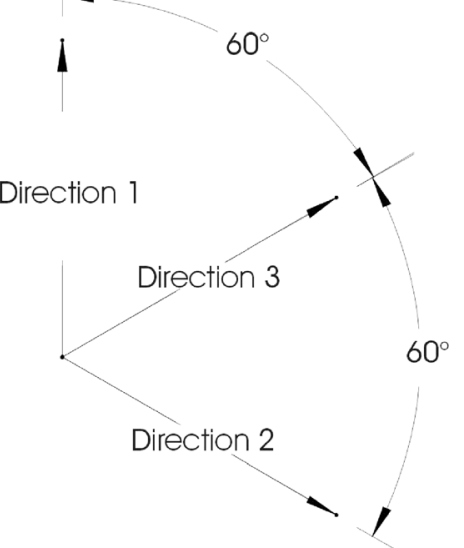

(d)

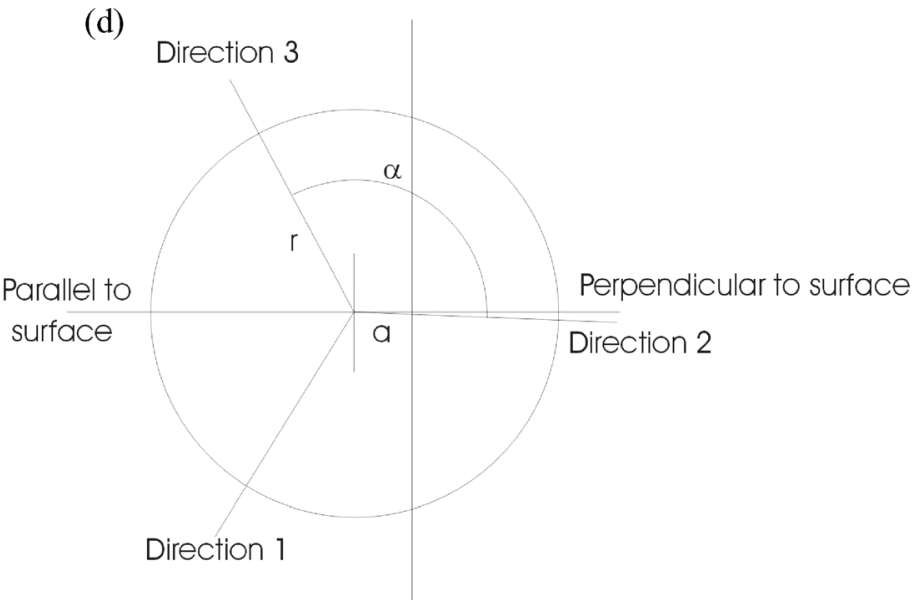

Fig. 1 The induction-hardened gear tooth, showing the surface-treated region and the measurement directions in relation to the gear, represented on a Mohr's circle for a region close to the surface

Straightforward use of trigonometric identities allows the equations for the strains $\varepsilon_{1}, \varepsilon_{2}$ and $\varepsilon_{3}$ to be manipulated together to find the three unknowns $\alpha, a$ and $r$ as follows:

$$
\begin{aligned}
& a=\frac{\varepsilon_{1}+\varepsilon_{2}+\varepsilon_{3}}{3} \\
& r \cos (2 \alpha)=\frac{2 \varepsilon_{1}-\varepsilon_{2}-\varepsilon_{3}}{3} \\
& r \sin (2 \alpha)=\frac{\varepsilon_{3}-\varepsilon_{2}}{\sqrt{3}} \\
& r=\sqrt{[r \cos (2 \alpha)]^{2}+[r \sin (2 \alpha)]^{2}} \\
& \alpha=\frac{1}{2} \arctan \left[\frac{r \sin (2 \alpha)}{r \cos (2 \alpha)}\right]
\end{aligned}
$$

Once these parameters are known, the direct strain at any direction $\theta$ with respect to strain direction 1 is simply found from

$$
\varepsilon(\theta)=a+r \cos (2 \alpha+2 \theta)
$$

A schematic representation of the three strains on the Mohr's circle is shown in Fig. 1 for a region close to the surface of the gear.

S05801 C IMechE 2002
Now let a global Cartesian system of $x-y$ coordinates be introduced. The above procedure allows the principal strain values and orientation to be determined at any point. Given knowledge of the parameters $a, r$ and $\alpha$, the Cartesian strain components $\varepsilon_{x x}$ and $\varepsilon_{y y}$ can now be evaluated.

Under the assumptions of plane strain, the stresses in a homogeneous and isotropic material are found from the standard equations

$$
\begin{aligned}
\sigma_{x x} & =\frac{E}{(1+v)(1-2 v)}\left[(1-v) \varepsilon_{x x}+v \varepsilon_{y y}\right] \\
\sigma_{y y} & =\frac{E}{(1+v)(1-2 v)}\left[(1-v) \varepsilon_{y y}+v \varepsilon_{x x}\right]
\end{aligned}
$$

The implementation of this calculation procedure in order to obtain residual stress maps in the gear tooth is described in the following section.

\section{RESULTS AND DISCUSSION}

The measured strains in the three chosen orientations are presented in Figs 2 to 4. It is clear that a strain increase towards the surface-treated region can be observed in all

JOURNAL OF STRAIN ANALYSIS VOL 37 NO 4 

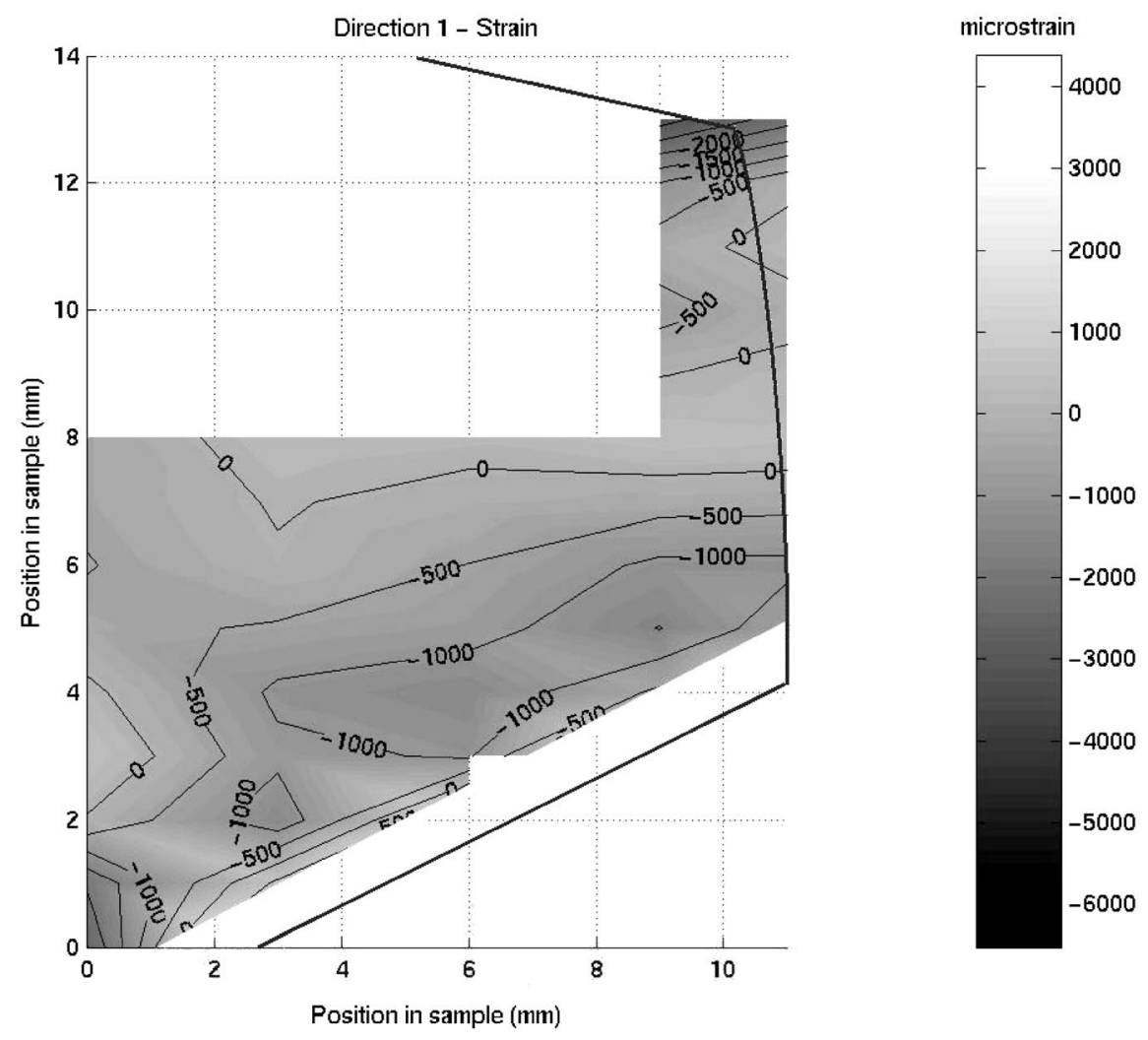

Fig. 2 Strain measured in direction 1, as shown in Fig. 1
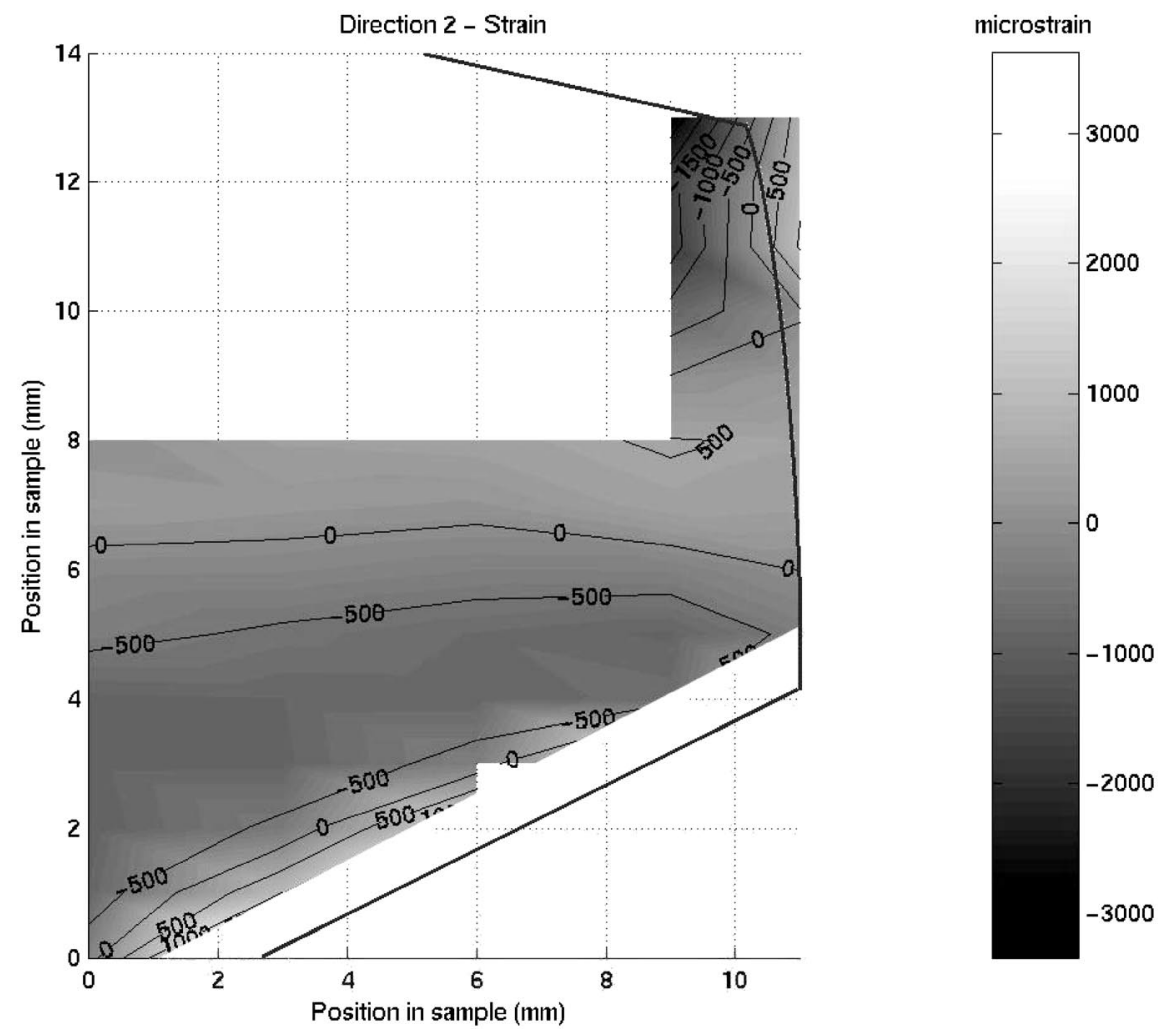

Fig. 3 Strain measured in direction 2, as shown in Fig. 1 

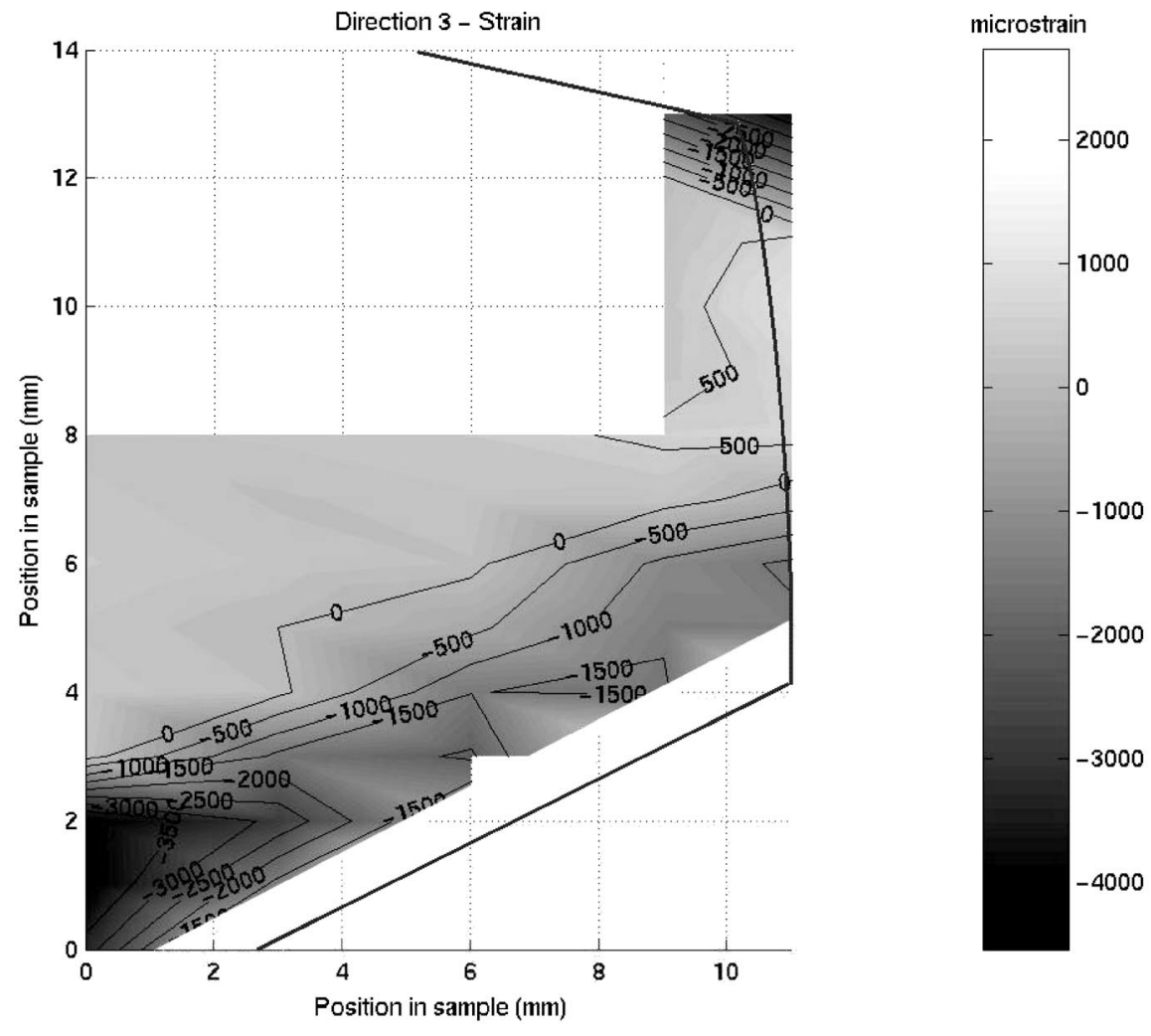

Fig. 4 Strain measured in direction 3, as shown in Fig. 1

three cases. However, it is also apparent that the effect appears to be more prominent in some directions than in others.

Direct measurement of strain parallel to the gear tooth surface was impracticably expensive in terms of beam time requirement in the present experiment. However, the data from the three strain maps for directions 1, 2 and 3 can be combined in order to determine the strains parallel and perpendicular to the gear tooth surface. These maps are presented in Figs 5 and 6 respectively. The three strain maps can also be used to determine the principal strain orientations and magnitudes. The results are illustrated in Figure 7.

Since the length of the gear tooth in the direction perpendicular to the section shown in Fig. 1 significantly exceeds its in-plane dimensions, and the treatment conditions were uniform along the length, the stress state can be assumed to correspond to that of plane strain. Using these assumptions, the stresses in the gear were calculated.

The principal stresses in the gear tooth were calculated, and the most tensile principal stress is shown in Fig. 8, in the orientations indicated in the map of Fig. 7. The map of the stress component acting parallel to the tooth surface is shown in Fig. 9. An area of compressive stress of approximately $-600 \mathrm{MPa}$ is clearly delineated, with the highest magnitude exceeding $1000 \mathrm{MPa}$. From the stress reconstruction it is clear that surface treatment has resulted in a band of compressive stress acting parallel to the gear surface to the depth up to $2-3 \mathrm{~mm}$. Note that the direction of principal compressive stress following heat treatment also runs parallel to the surface, as illustrated in Fig. 7.

\section{CONCLUSION}

The results of neutron diffraction strain mapping in an induction-hardened gear tooth are presented. It is demonstrated how neutron diffraction measurement of strains in three directions can be used to reduce the measurement time required, by using the minimum of three orientations each requiring shorter beam path lengths. The information can then be used to construct the complete description of the stress state in terms of the principal stress values and orientations in the mapped region, and to build up maps of in-plane strains and stresses in arbitrary orientations.

\section{ACKNOWLEDGEMENT}

The authors would like to thank the EU-Training and Mobility of Researchers Scheme (EU-TMR) for funding their access to the neutron scattering facilities at Laboratoire Léon Brillouin, Saclay.

JOURNAL OF STRAIN ANALYSIS VOL 37 NO 4 

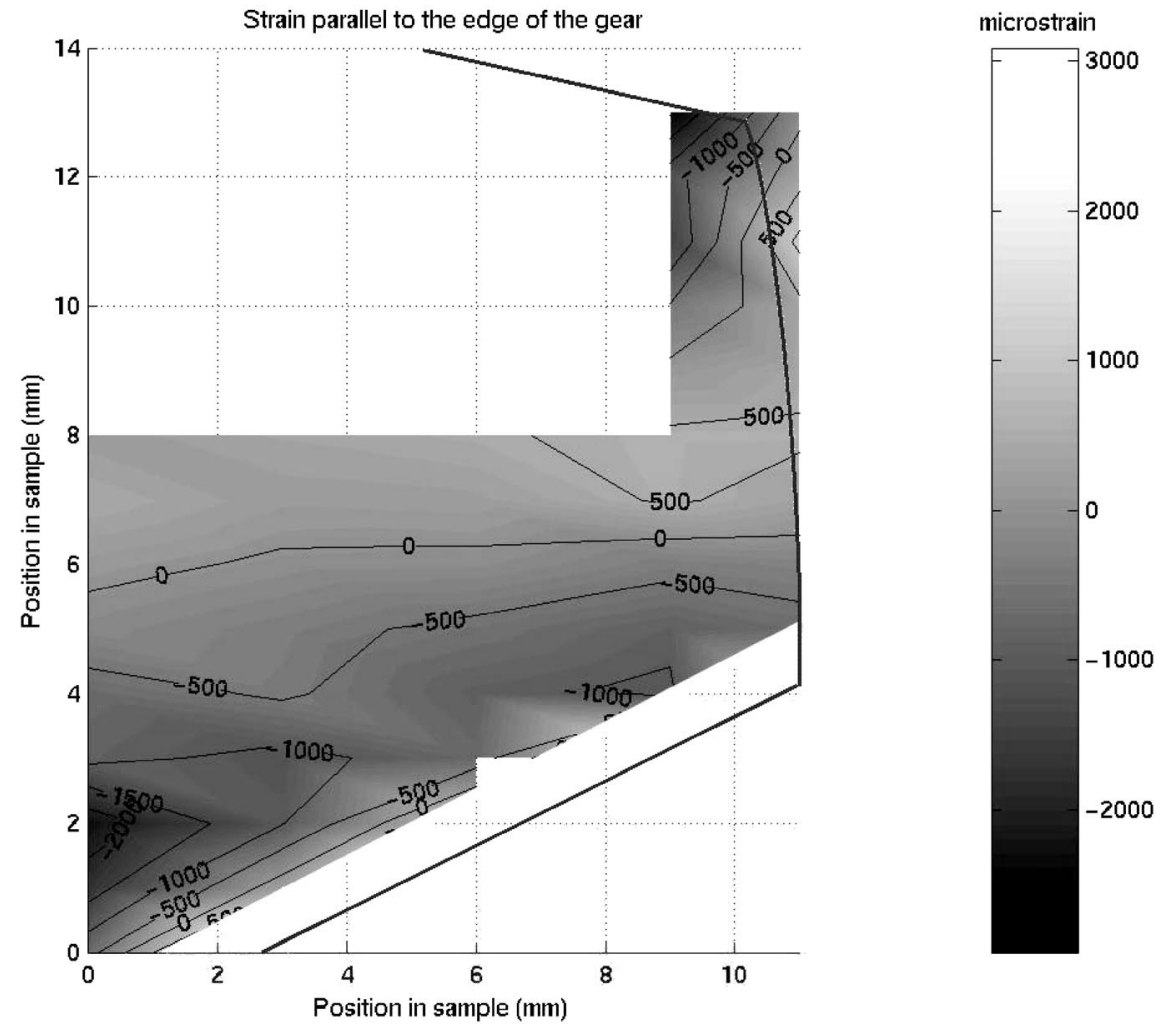

Fig. 5 Strain measured parallel to the edge of the tooth

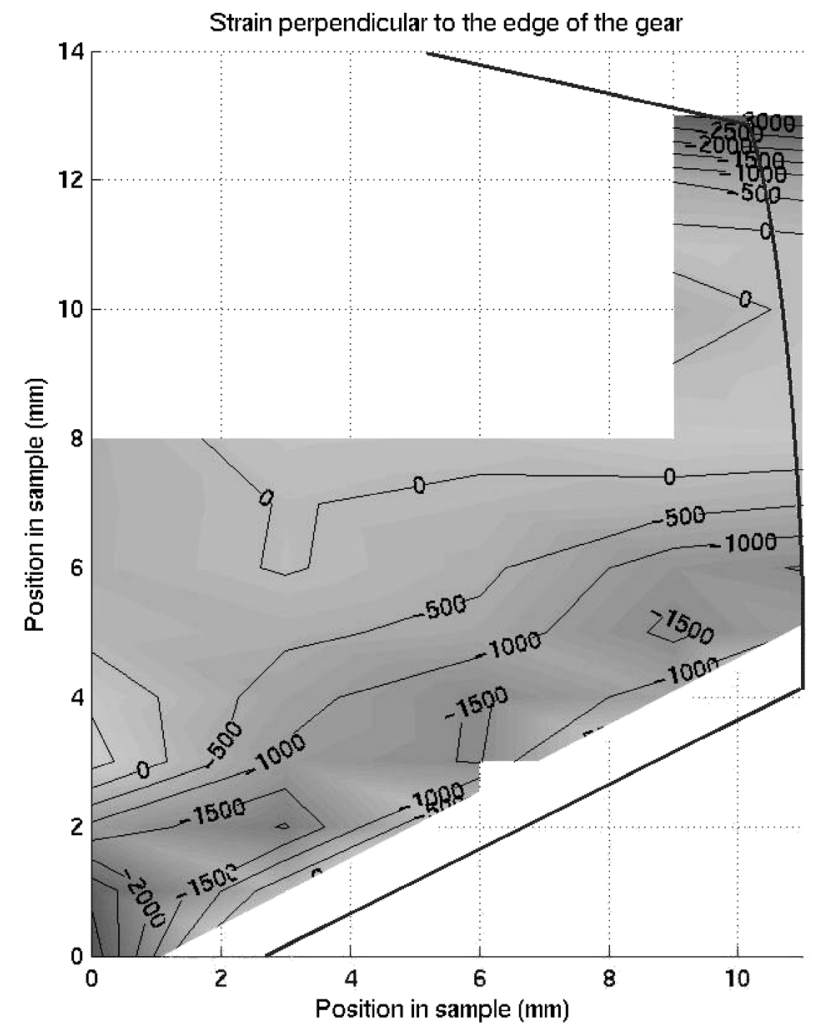

microstrain

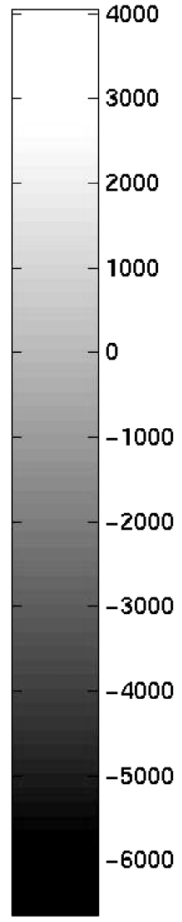

Fig. 6 Strain measured perpendicular to the edge of the tooth 


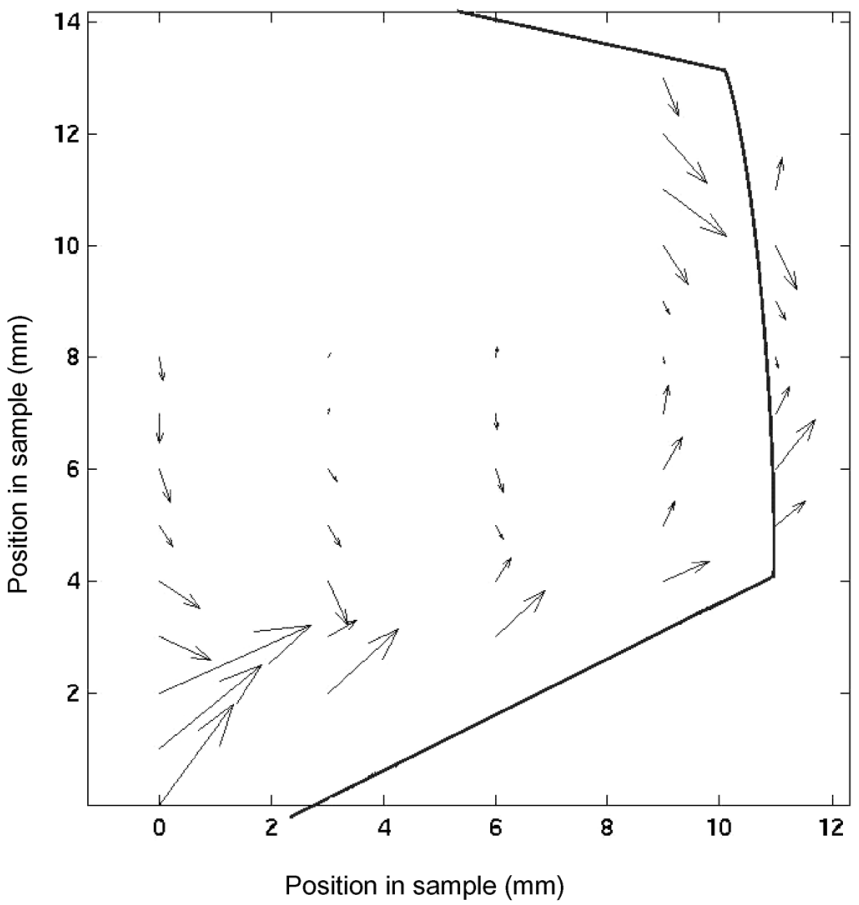

Fig. 7 Principal strain directions, shown overlying the gear tooth

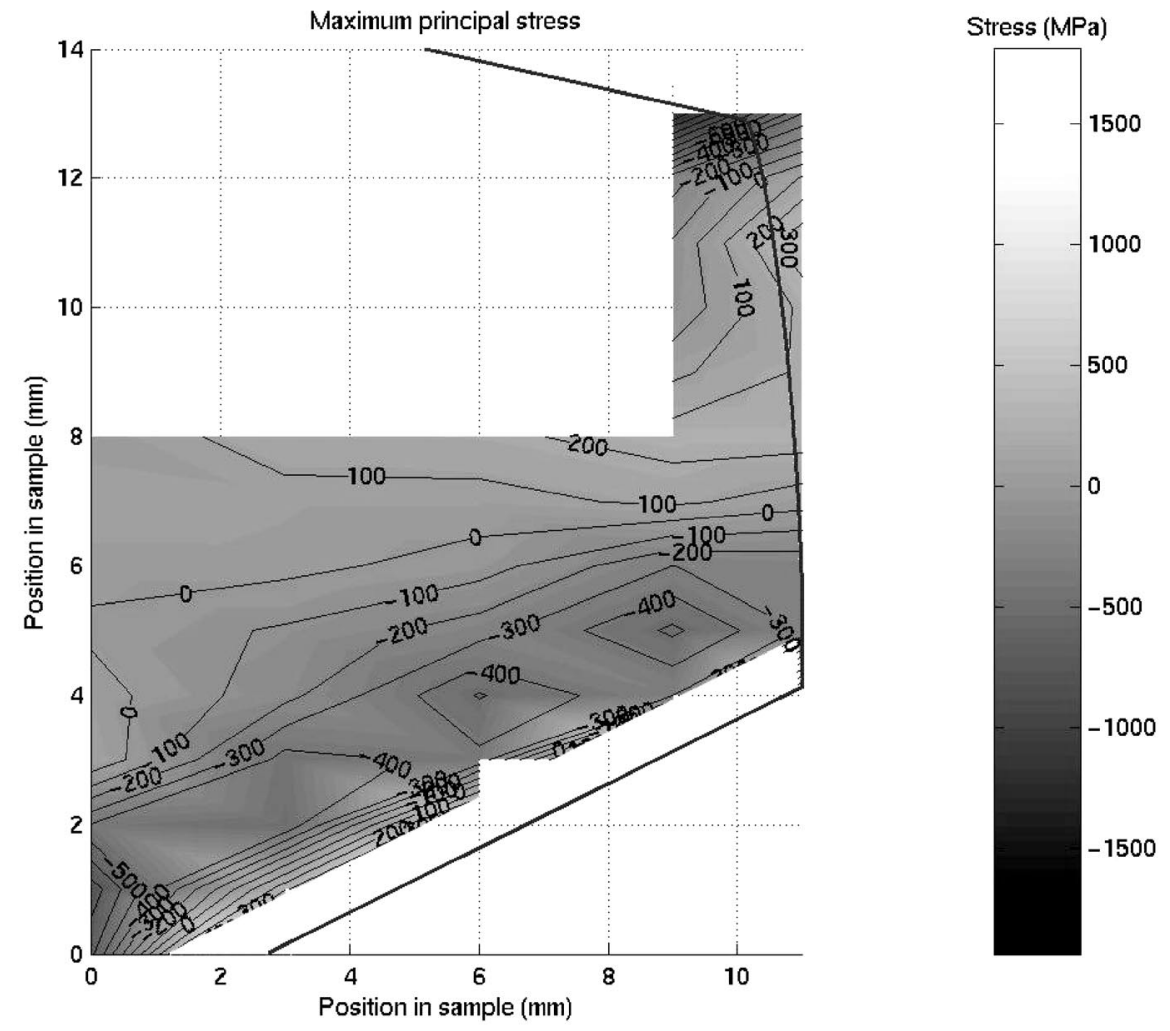

Fig. 8 Principal stress in the gear tooth, calculated assuming plane strain conditions 

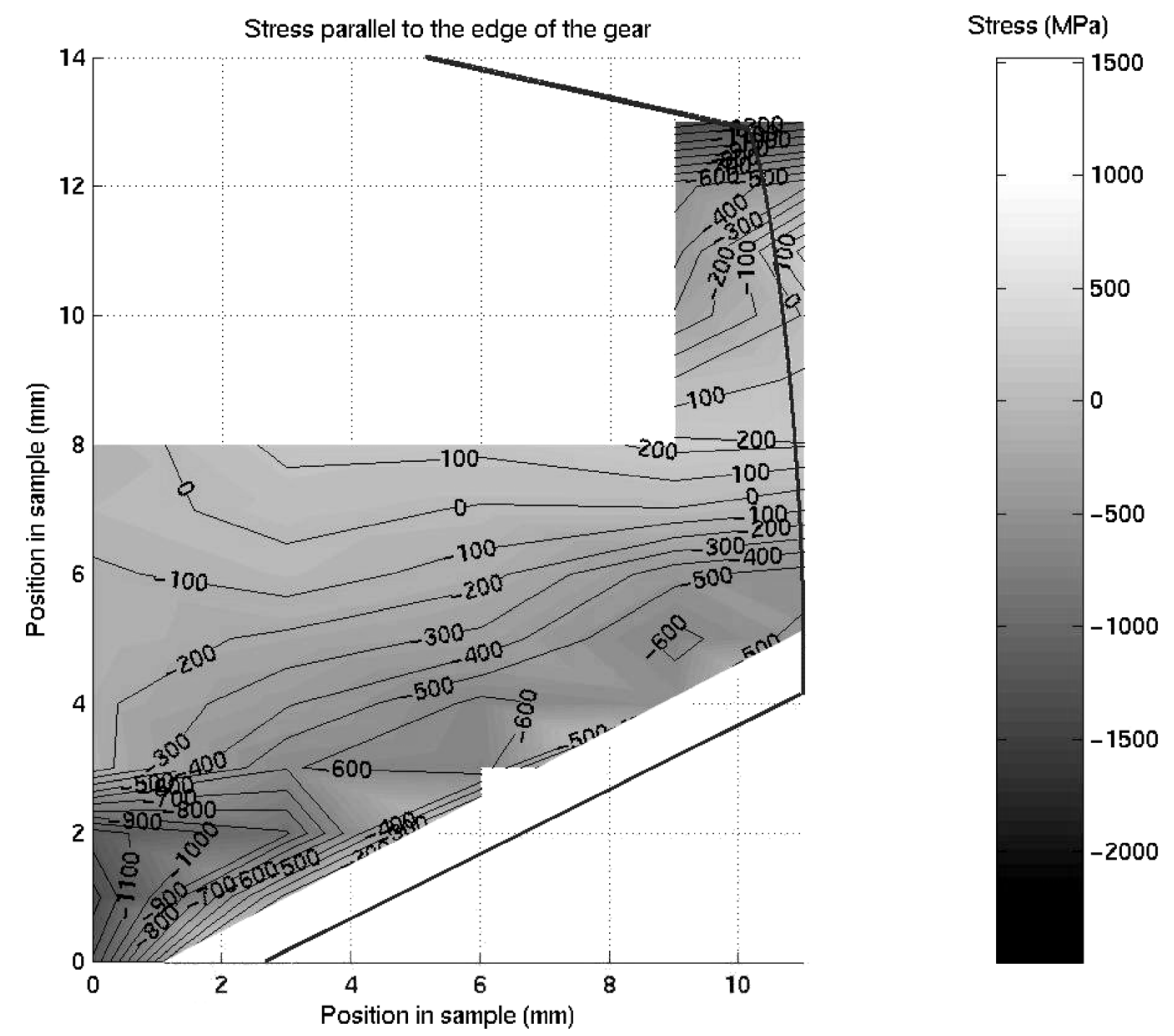

Fig. 9 Stress parallel to the edge of the gear tooth, calculated assuming plane strain conditions

\section{REFERENCES}

1 Shaw, B. A., Korsunsky, A. M. and Evans, J. T. Surface treatment and residual stress effects on the fatigue strength of carburised gears. Presented at the 12th European Conference on Fracture (ECF-12), Sheffield, 14-18 September 1998.
2 Cullity, B. D. Elements of X-ray Diffraction, 1978 (AddisonWesley, London).

3 Korsunsky, A. M., Wells, K. E. and Shaw, B. A. A comparative study of diffraction methods for strain measurement in a particulate MMC. Mater. Sci. Forum, 2000, 347349, 504-509.

4 Gere, J. M. and Timoshenko, S. P. Mechanics of Materials, 1990 (PWS-Kent, Boston, Massachusetts). 